\title{
Efficacy and safety of gonadotropin-releasing hormone agonists used in the treatment of prostate cancer
}

This article was published in the following Dove Press journal:

Drug, Healthcare and Patient Safety

2I November 2011

Number of times this article has been viewed

\author{
Seungtaek Choi \\ Andrew K Lee \\ Department of Radiation Oncology, \\ MD Anderson Cancer Center, \\ Houston, TX, USA
}

\begin{abstract}
Androgen deprivation therapy (ADT) is the most effective systemic treatment for prostate cancer. ADT has been shown to have a high rate of response and to improve overall survival in patients with metastatic prostate cancer. In addition, multiple studies have shown that adding ADT to external beam radiation therapy leads to improvement in cure rates and overall survival in prostate cancer patients. The most commonly used ADT is gonadotropin-releasing hormone $(\mathrm{GnRH})$ agonist therapy. Although GnRH agonist therapy has significant benefits for patients with prostate cancer, it has also been shown to have significant side effects, including fatigue, hot flashes, decreased libido, decreased quality of life, obesity, diabetes mellitus, coronary artery disease, decreased bone mineral density, and increased risk of fractures. Therefore, it is crucial that the benefits of ADT be weighed against its potential adverse effects before its use. Keywords: androgen deprivation therapy, gonadotropin-releasing hormone agonists, prostate cancer
\end{abstract}

\section{Introduction}

Prostate cancer is the most common solid malignancy in men, with 217,730 estimated new cases in 2010. ${ }^{1}$ Prostate cancer is the second most common cause of cancer death in men, only surpassed by lung cancer, with 32,050 estimated deaths in 2010. The 5-year overall survival for patients with localized or regional prostate cancer approaches $100 \%$. Even patients with metastatic prostate cancer at diagnosis have a 5-year overall survival of $31 \%{ }^{1}$ There are multiple modalities available for the treatment of prostate cancer, including surgery, radiation therapy, and androgen deprivation therapy (ADT).

ADT is the most effective systemic treatment for prostate cancer, and has been shown to have a response rate of approximately $85 \%$ in patients with metastatic disease. ${ }^{2,3}$ It has been shown to decrease pain in patients with bone metastases and lead to improvement in overall survival. ${ }^{4}$ ADT has also been shown to improve diseasefree survival and overall survival in patients with high-risk/locally advanced prostate cancer when given with radiation therapy. Furthermore, ADT increases overall survival in patients with lymph node-positive disease when given in an adjuvant manner after prostatectomy and lymph node dissection. ${ }^{5}$ ADT can be used to decrease the size of the prostate in patients whose prostate is too large for prostate seed implant or to decrease the radiation dose to the normal tissues around the prostate.

Although ADT has significant benefits for patients with prostate cancer, it has also been shown to have significant side effects, including fatigue, hot flashes, decreased libido, decreased quality of life, obesity, diabetes mellitus, coronary artery disease, decreased bone mineral density, and increased risk of fractures. Therefore, it is essential
Correspondence: Seungtaek Choi I840 Old Spanish Trail, Unit II50, Houston, TX 77054, USA

Tel +l 7I3 5632288

Fax + | 713563 152|

Email stchoi@mdanderson.org 
that one weigh the benefits of ADT against the potential side effects before use in patients with prostate cancer.

\section{Androgen deprivation therapy Mechanism of action of $\mathrm{GnRH}$ agonists}

Testosterone produced by the testes is the main source of circulating androgens in males. A total serum testosterone level of $300 \mathrm{ng} / \mathrm{dL}$ is usually considered to be the lower limit of normal, because patients with testosterone levels below this threshold have been shown to have a greater likelihood of having symptoms. ${ }^{6,7}$ Testosterone levels can be reduced either through the use of medications or by surgical orchiectomy. Medical castration is usually favored due to the irreversible nature of surgical orchiectomy; however, orchiectomy costs significantly less than long-term medical castration. ${ }^{8}$ A castrate level of testosterone is usually defined as $50 \mathrm{ng} / \mathrm{dL}$ or less.

Androgen deprivation is often achieved by suppression of the release of luteinizing hormone from the anterior pituitary. Gonadotropin-releasing hormone $(\mathrm{GnRH})$ is a decapeptide hormone synthesized in the hypothalamus. ${ }^{9}$ When GnRH is released in a pulsatile fashion, it induces the anterior pituitary gland to release luteinizing hormone (Figure 1). Luteinizing hormone induces Leydig cells in the testes to produce testosterone. GnRH agonists mimic GnRH and shuts down luteinizing hormone production by continuous pituitary stimulation, overcoming the endogenous pulsatile

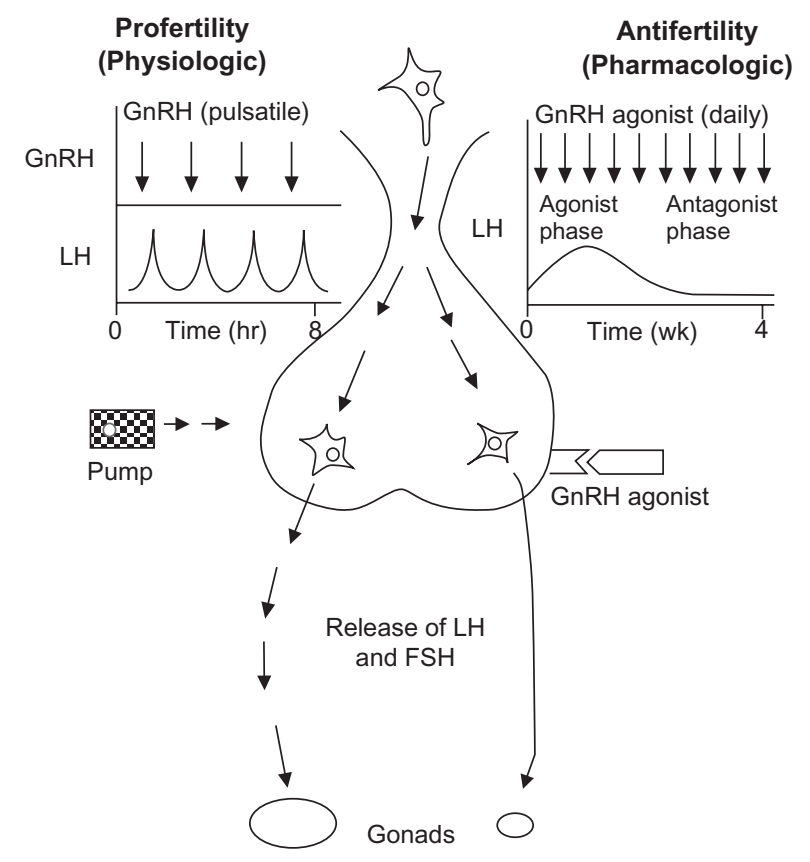

Figure I Mechanism of action of gonadotropin-releasing hormone agonists. ${ }^{9}$ Adapted from Conn and Crowley, used with permission.

Abbreviations: $\mathrm{GnRH}$, gonadotropin-releasing hormone; $\mathrm{FSH}$, follicle-stimulating hormone; LH, luteinizing hormone.
GnRH release. GnRH agonists are the most commonly used medications for ADT in the treatment of prostate cancer patients. There are several medications in the GnRH agonist family (Table 1). The two most commonly used medications are leuprolide (also known as Lupron ${ }^{\circledR}$ and Eligard ${ }^{\circledR}$ ) and goserelin $\left(\right.$ Zoladex $\left.^{\circledR}\right)$. Leuprolide is usually given as an intramuscular injection (in the gluteal region) whereas goserelin is usually given as a subcutaneous injection (usually in the abdomen).

When GnRH agonists are given, there can be an initial rise in the level of testosterone (known as the "flare"). Such an increase can stimulate prostate cancer cells to grow and cause patients to have worsening symptoms. For instance, patients with bone metastases could have worsening of their pain. Therefore, a nonsteroidal androgen receptor antagonist is often given before the GnRH agonist to block the effect of the rise in testosterone. A GnRH antagonist offers an alternative method of achieving androgen deprivation without the initial surge in testosterone. A randomized Phase III trial tested degarelix, a GnRH antagonist, $240 \mathrm{mg}$ given initially, followed by either $80 \mathrm{mg}$ or $160 \mathrm{mg}$ a month subcutaneously against leuprolide $7.5 \mathrm{mg}$ given initially, followed by $7.5 \mathrm{mg}$ monthly. ${ }^{10}$ By day 3, the median testosterone levels were $24 \mathrm{ng} / \mathrm{dL}$ and $26 \mathrm{ng} / \mathrm{dL}$ for the patients in the degarelix 240/80 $\mathrm{mg}$ and 240/160 mg arms, respectively. In contrast, the median testosterone level rose from $384.4 \mathrm{ng} / \mathrm{dL}$ to $630 \mathrm{ng} / \mathrm{dL}$ for the patients in the leuprolide arm. The prostate

Table I Gonadotropin-releasing hormone agonists used in treatment of prostate cancer

\begin{tabular}{|c|c|c|}
\hline Generic name & Brand name & Dosage \\
\hline \multirow[t]{11}{*}{ Leuprolide acetate } & Lupron $^{\circledast}$ depot & Intramuscular injection \\
\hline & & I month: 7.5 mg \\
\hline & & 3 months: $22.5 \mathrm{mg}$ \\
\hline & & 4 months: $30 \mathrm{mg}$ \\
\hline & Eligard $^{\circledR}$ & Subcutaneous injection \\
\hline & & I month: $7.5 \mathrm{mg}$ \\
\hline & & 3 months: $22.5 \mathrm{mg}$ \\
\hline & & 4 months: $30 \mathrm{mg}$ \\
\hline & & 6 months: $45 \mathrm{mg}$ \\
\hline & Viadur $^{\circledR}$ & Intradermal implant \\
\hline & & 12 months: 65 mg \\
\hline \multirow[t]{3}{*}{ Goserelin acetate } & Zoladex $^{\circledR}$ & Subcutaneous injection \\
\hline & & I month: $7.5 \mathrm{mg}$ \\
\hline & & 3 months: $22.5 \mathrm{mg}$ \\
\hline \multirow[t]{6}{*}{ Triptorelin pamoate } & Trelstar $^{\circledast}$ depot & Intramuscular injection \\
\hline & & I month: 3.75 mg \\
\hline & Trelstar LA & Intramuscular injection \\
\hline & & 3 months: $11.25 \mathrm{mg}$ \\
\hline & Trelstar & Intramuscular injection \\
\hline & & 6 months: $22.5 \mathrm{mg}$ \\
\hline \multirow[t]{2}{*}{ Buserelin acetate } & Suprefact ${ }^{\circledR}$ & Subcutaneous injection \\
\hline & & Every 8 hours: $0.5 \mathrm{mg}$ \\
\hline
\end{tabular}


serum antigen (PSA) levels 14 days after the injection were also lower for the patients in the degarelix arms, once again showing a faster decline in PSA levels. By day 35, there was no statistically significant difference between the degarelix and leuprolide arms. Testosterone suppression (defined as levels $<50 \mathrm{ng} / \mathrm{mL}$ ) was achieved in $97.2 \%, 98.3 \%$, and $96.4 \%$ of patients in the degarelix $240 / 80 \mathrm{mg}$, degarelix $240 / 160 \mathrm{mg}$, and leuprolide arms, respectively. ${ }^{10}$

\section{Other hormonal agents}

GnRH agonists may not be able to block testosterone production completely in males, because the adrenal glands can also produce androgens. Ketoconazole, an imidazole antifungal that inhibits cytochrome P450 (CYP) enzymes, including 17-alpha-hydroxylase/17,20-lyase (CYP17A1), is very effective in decreasing testosterone production in the adrenal gland. However, this medication must be used with care and these patients require careful monitoring. Abiraterone acetate is a more specific and potent inhibitor of CYP17A1, which has been shown to improve overall survival in patients with metastatic castrate-resistant prostate cancer. A randomized trial by de Bono et al showed that treatment with abiraterone significantly improved median overall survival from 10.9 months to 14.8 months when compared with treatment with placebo (hazard ratio [HR]: 0.65 , $P<0.001) .{ }^{11}$ All secondary endpoints, including time to PSA progression (10.2 vs 6.6 months, $P<0.001)$, progressionfree survival (5.6 months vs 3.6 months, $P<0.001$ ), and PSA response rate $(29 \%$ vs $6 \%, P<0.001)$ were improved in patients receiving abiraterone.

A decline in serum androgens may not lead to a similar decrease in intraprostatic androgens. Testosterone is rapidly reduced by 5 -alpha-reductase to dihydrotestosterone in the prostate. Despite the $94 \%$ decline in serum testosterone with ADT, intraprostatic concentrations of testosterone and dihydrotestosterone decline by only $70 \%-80 \% .^{12} 5$-alphareductase inhibitors such as flutamide or dutasteride can be used to decrease the intraprostatic conversion from testosterone to dihydrotestosterone.

As stated earlier, androgen receptor antagonists can be used with GnRH agonists to decrease the effect of the testosterone surge. They can also be used in patients with castrate-resistant prostate cancer. Androgen receptor antagonists can be classified into two classes, ie, steroidal and nonsteroidal. Cyproterone acetate is a steroidal antiandrogen, which also has weak progestational and glucocorticoid activity. Nonsteroidal antiandrogens include bicalutamide, flutamide, and nilutamide. Of these, bicalutamide has the longest half-life and binds to the androgen receptor with the highest affinity. ${ }^{13}$ However, nonsteroidal antiandrogens can have agonist activity on the androgen receptor. MDV3100 is a new androgen receptor antagonist, which binds to the androgen receptor with 5-8-fold higher affinity than bicalutamide, inhibits androgen receptor nuclear translocation, and has reduced agonist activity. ${ }^{14}$ MDV3100 is also currently being tested in castrate-resistant prostate cancer patients.

\section{Use of GnRH agonists in prostate cancer}

Treatment of prostate cancer depends on the clinical stage, Gleason score, and PSA level at presentation. Based on these factors, prostate cancer can be divided into localized, locally advanced, and metastatic disease. Localized prostate cancer can usually be treated with definitive single-modality treatments, such as surgery or radiotherapy. Although ADT is not considered to be a definitive treatment, it can be used in conjunction with radiotherapy in select situations to improve clinical outcomes. For instance, patients with higher presenting Gleason scores and PSA levels may benefit from the addition of hormone therapy to radiotherapy. The benefit of adding hormone therapy to surgery in patients with localized prostate cancer has not yet been seen in randomized studies. Locally advanced prostate cancer usually requires combined modality treatment of either surgery and adjuvant radiotherapy or radiotherapy with ADT. When patients have recurrence of prostate cancer after definitive treatment, ADT can be used for salvage treatment. For patients with metastatic prostate cancer (with either bone or lymph node metastasis), ADT is usually the first-line treatment.

ADT is often used in the treatment in patients who have a recurrence after definitive local treatment. Antiandrogen therapy has been shown to improve clinical outcome when added to salvage radiotherapy (in patients with a rising PSA after surgery with likely recurrence in the surgical bed). The Radiation Therapy Oncology Group (RTOG) 96-01 trial randomized 771 patients with a rising PSA after prostatectomy (pT2, N0 or pT3, N0) to either radiotherapy alone or radiotherapy with 24 months of bicalutamide. ${ }^{15}$ Seven-year freedom from PSA progression was significantly improved in the radiotherapy + bicalutamide arm (57\% vs $40 \%, P<0.0001)$. The seven-year incidence of metastatic disease was also decreased with the addition of bicalutamide (7.4\% vs $12.6 \%, P=0.041)$. ADT is usually the recommended treatment in patients with a rising PSA and likely metastatic disease. Until recently, the standard of care was to treat these patients with ADT given continuously. A recent study by 
Klotz et al showed that there is no significant difference in overall survival or quality of life between giving intermittent androgen suppression and continuous androgen deprivation. The median survival was 8.8 years in the intermittent androgen suppression arm vs 9.1 years in the continuous androgen deprivation arm (HR: 1.02, $P=0.009) .{ }^{16}$ Furthermore, there was an increased time to castration resistance with intermittent androgen suppression (10 years vs 9.8 years, HR: $0.80, P=0.024)$. Patients undergoing intermittent androgen suppression were on medication for $27 \%$ of the time, which could represent significant cost savings in terms of medication costs. At this time, treatment with intermittent ADT should be considered to be the new standard of care for these patients.

Patients with metastatic disease have a high chance of responding to ADT using GnRH agonists. A study by the Leuprolide Study Group showed an objective response of $86 \%$ in patients with metastatic prostate cancer receiving leuprolide. ${ }^{2}$ A similar study by Volzegang et al showed an objective response of $82 \%$ for patients with metastatic prostate cancer receiving goserelin. ${ }^{3}$ ADT has been shown to improve clinical outcome significantly when used in patients with locally advanced or metastatic disease. A study by the Medical Research Council randomized patients with locally advanced or asymptomatic metastatic prostate cancer to either immediate ADT using a GnRH agonist or orchiectomy or observation until ADT was clinically indicated. ${ }^{4}$ Pathologic fracture, spinal cord compression, ureteral obstruction, and extraskeletal metastases were twice as common in patients who did not receive immediate ADT. In total, 361 patients died in the deferred ADT arm compared with 328 in the immediate ADT arm $(P=0.02)$. In total, 257 patients died from prostate cancer in the deferred ADT arm compared with 203 in the immediate ADT $\operatorname{arm}(P=0.001){ }^{4}$

\section{$\mathrm{GnRH}$ agonist given with radiotherapy}

There have been several randomized trials showing the benefit of adding 3-6 months of ADT to radiotherapy. These trials are summarized in Table 2. D'Amico et al randomized patients

Table 2 Summary of studies showing benefit of short-course androgen deprivation therapy

\begin{tabular}{|c|c|c|c|c|}
\hline Study & Entry criteria & Treatments & Patients (n) & Results \\
\hline \multirow[t]{4}{*}{$D^{\prime} A m i c o$ et $a^{17}$} & Gleason 7-10, & EBRT alone & 104 & Median follow-up: 7.6 years \\
\hline & $\mathrm{PSA}>10 \mathrm{ng} / \mathrm{mL}$ & EBRT +6 months of leuprolide & 102 & ADT improved: \\
\hline & (maximum $40 \mathrm{ng} / \mathrm{mL}$ ), & or goserelin and flutamide & & OS $(74 \%$ vs $61 \%, P=0.01)$ \\
\hline & ECE or SVI on MRI & & & PCSM (HR: 4.I, $P=0.0 \mathrm{I})$ \\
\hline \multirow[t]{6}{*}{ RTOG $86-10^{18}$} & Stage T2-T4 & EBRT alone & 232 & Median follow-up: II.9 years \\
\hline & $5 \times 5 \mathrm{~cm}$ tumors & EBRT +4 months of goserelin & 224 & ADT improved at 10 years: \\
\hline & & and flutamide & & $\operatorname{PCSM}(23.3 \%$ vs $35.6 \%, P=0.01)$ \\
\hline & & & & $\mathrm{BF}(65.1 \%$ vs $80.0 \%, P<0.0001)$ \\
\hline & & & & DFS ( $11.2 \%$ vs $3.4 \%, P<0.0001)$ \\
\hline & & & & $\operatorname{DMR}(34.9 \%$ vs $46.9 \%, P=0.006)$ \\
\hline \multirow[t]{8}{*}{ RTOG 94-08 19} & Stage TIb-T2b & EBRT alone & 992 & Median follow-up: 9.1 years \\
\hline & $\mathrm{PSA} \leq 20 \mathrm{ng} / \mathrm{mL}$ & EBRT +4 months of leuprolide & 987 & ADT improved at 10 years: \\
\hline & & or goserelin and flutamide & & OS $(62 \%$ vs $57 \%, P=0.03)$ \\
\hline & & & & $\operatorname{PCSM}(4 \%$ vs $8 \%, P=0.001)$ \\
\hline & & & & BF $(26 \%$ vs $41 \%, P<0.001)$ \\
\hline & & & & DMR ( $6 \%$ vs $8 \%, P=0.04)$ \\
\hline & & & & Rate of positive biopsies at \\
\hline & & & & 2 years $(20 \%$ vs $39 \%, P<0.00 I)$ \\
\hline \multirow[t]{10}{*}{ TROG $96.0 \mathrm{I}^{20}$} & Stage $\mathrm{T} 2 \mathrm{~b}-\mathrm{T} 4$ & EBRT alone & 270 & Median follow-up: 10.6 years \\
\hline & & EBRT +3 months of goserelin & 265 & 3 months of ADT improved: \\
\hline & & and flutamide & & BF (HR: $0.72, P=0.003)$ \\
\hline & & & & DFS (HR: $0.63, P<0.000 \mathrm{I})$ \\
\hline & & EBRT +6 months of goserelin & 267 & 6 months of ADT improved: \\
\hline & & and flutamide & & BF (HR: $0.57, P<0.000 \mathrm{I})$ \\
\hline & & & & DFS (HR: $0.5 \mathrm{I}, P<0.000 \mathrm{I})$ \\
\hline & & & & DMR (HR: $0.49, P=0.00 \mathrm{I})$ \\
\hline & & & & PCSM (HR: $0.49, P=0.0008)$ \\
\hline & & & & ACM (HR: $0.63, P=0.0008)$ \\
\hline
\end{tabular}

Abbreviations: PSA, prostate specific antigen; PCSM, prostate cancer-specific mortality; ECE, extracapsular extension; RTOG, Radiation Therapy Oncology Group; SVI, seminal vesicle invasion; HR, hazard ratio; MRI, magnetic resonance imaging; BF, biochemical failure; EBRT, external beam radiation therapy; DFS, disease-free survival; ADT, androgen deprivation therapy; OS, overall survival; DMR, distant metastases rate; ACM, all-cause mortality; TROG, Trans-Tasman Radiation Oncology Group. 
with localized, unfavorable risk prostate cancer to either external beam radiation therapy (EBRT) with 6 months of ADT or to EBRT alone. ${ }^{17}$ ADT consisted of a GnRH agonist (either leuprolide or goserelin) and a nonsteroidal antiandrogen (flutamide) starting 2 months before radiotherapy. ADT was continued during the 2 months of radiotherapy and continued for 2 months afterwards. Unfavorable risk was defined as a PSA level more than $10 \mathrm{ng} / \mathrm{mL}$ (maximum $40 \mathrm{ng} / \mathrm{mL}$ ), Gleason score 7 to 10 , and/or evidence of extracapsular extension and/or seminal vesicle invasion on endorectal magnetic resonance imaging. After a median follow-up of 7.6 years, patients who were randomized to receive radiotherapy alone had a significantly increased risk of death compared with patients who were randomized to receive ADT with radiotherapy (HR: $1.8, P=0.01) .{ }^{17}$ When a subgroup analysis was performed, the benefit of adding ADT was significantly dependent on the presence of medical comorbidities, as determined by a 27-item comorbidity index (Adult Comorbidity Evaluation 27). The patients who had no or minimal comorbidity had a significant improvement in outcome with the addition of ADT (HR: 4.2, $P<0.001)$. When patients had moderate or severe comorbidity, there was no significant improvement in outcome with the addition of ADT (HR: $0.54, P=0.08$ ).

RTOG 86-10 compared patients with bulky tumors $(5 \times 5 \mathrm{~cm}$, stage T2-T4) who received either EBRT with 4 months of ADT or EBRT alone. ${ }^{18}$ ADT consisted of goserelin $3.6 \mathrm{mg}$ every 4 weeks and flutamide $250 \mathrm{mg}$ three times per day for 2 months before and during radiotherapy. Although there was no overall survival benefit at 10 years between the EBRT and ADT group vs the EBRT only group (42.6\% vs $33.8 \%, P=0.12$ ), there was a significant improvement in prostate cancer-specific mortality at 10 years $(23.3 \%$ vs $35.6 \%, P=0.01) .{ }^{18}$ Furthermore, there was an improvement in the biochemical failure rate $(65.1 \%$ vs $80.0 \%, P<0.0001)$, disease-free survival ( $11.2 \%$ vs $3.4 \%, P<0.0001)$, and distant metastases rate $(34.9 \%$ vs $46.9 \%, P=0.006)$. There was also a significant delaying of distant failure. Approximately $40 \%$ of patients on EBRT alone had developed bone metastases by 5 years. In comparison, it took 14 years for $40 \%$ of patients on the EBRT and ADT arm to develop bone metastases.

RTOG 94-08 randomized 1979 patients with stage $\mathrm{T} 1 \mathrm{~b}-\mathrm{T} 2 \mathrm{~b}$ and PSA $\leq 20 \mathrm{ng} / \mathrm{mL}$ to either radiotherapy alone or radiotherapy with 4 months of ADT with a GnRH agonist (either monthly goserelin or leuprolide) and flutamide $250 \mathrm{mg}$ three times per day. ADT was started two months before the radiotherapy. After a median follow-up of 9.1 years, patients who received ADT had significant improvement in 10-year overall survival (62\% vs $57 \%, P=0.03)$, disease-specific mortality ( $4 \%$ vs $8 \%, P=0.001)$, biochemical failure $(26 \%$ vs $41 \%, P<0.001)$, distant metastases ( $6 \%$ vs $8 \%, P=0.04)$, and the rate of positive findings on repeat prostate biopsy at 2 years $(20 \%$ vs $39 \%, P<0.001) .{ }^{19}$ These improvements in overall survival and disease-specific mortality were seen primarily among patients with intermediate-risk cancers, with no significant improvement seen in patients with lowrisk cancers. Acute and late radiation-induced toxic effects were similar between the two groups.

The Trans-Tasman Radiation Oncology Group (TROG) randomized 818 men with T2b, T2c, T3, or T4 prostate cancer to receive radiotherapy alone, radiotherapy plus 3 months of ADT, or radiotherapy plus 6 months of ADT. The ADT consisted of goserelin $3.6 \mathrm{mg}$ given monthly and flutamide $250 \mathrm{mg}$ taken three times per day. ADT was started 2 months before radiotherapy for the 3-month group and 5 months before the radiotherapy for the 6-month group. After a median follow-up of 10.6 years, the addition of 3 months of ADT to radiotherapy decreased the rates of PSA progression (HR: $0.72, P=0.003$ ) and local progression (HR: 0.49, $P=0.0005$ ), and improved event-free survival (HR: 0.63, $P<0.0001) .{ }^{20}$ Six months of ADT added to radiotherapy also reduced PSA progression (HR: 0.57, $P<0.0001$ ), local progression (HR: $0.45, P=0.0001$ ), and improved event-free survival (HR: $0.51, P<0.0001$ ). Three months of ADT had no effect on distant progression (HR: 0.89, $P=0.550$ ), prostate cancer-specific mortality (HR: 0.86 , $P=0.398$ ), or all-cause mortality (HR: $0.84, P=0.180)$. By contrast, 6 months of ADT decreased distant progression (HR: $0.49, P=0.001$ ), prostate cancer-specific mortality (HR: $0.49, P=0.0008$ ), or all-cause mortality (HR: 0.63 , $P=0.0008)$. There was no increased morbidity with the addition of ADT to radiotherapy.

These studies show that addition of short-course ADT to EBRT decreases biochemical failure and can lead to improvement in disease-specific mortality and overall survival. However, depending on the aggressiveness and extent of the cancer, patients may require more than 3-6 months of hormone ablation therapy. Multiple randomized trials have shown the benefit of longer-term hormone ablation therapy with EBRT in patients with higher stage, Gleason scores, and/or PSA values. These trials are summarized in Table 3.

The European Organization for Research and Treatment of Cancer (EORTC) performed two studies evaluating the use of long-term ADT with radiotherapy in the treatment of prostate cancer. EORTC 22863 randomized patients with 
Table 3 Summary of studies showing benefit of long-term androgen deprivation therapy

\begin{tabular}{|c|c|c|c|c|}
\hline Study & Entry criteria & Treatments & Patients (n) & Results \\
\hline \multirow[t]{6}{*}{ EORTC $22863^{21}$} & WHO grade 3 & EBRT alone & 208 & Median follow-up: 9.1 years \\
\hline & Stage T3-T4 & $\mathrm{EBRT}+36$ months of goserelin & 207 & Long-term ADT improved at 10 years: \\
\hline & Lymph node-negative & and one month of cyproterone & & OS (58. $1 \%$ vs $39.8 \%, P=0.0004)$ \\
\hline & & & & $\operatorname{PCSM}(10.3 \%$ vs $30.4 \%, P<0.000 I)$ \\
\hline & & & & DFS $(47.7 \%$ vs $22.7 \%, P<0.000 I)$ \\
\hline & & & & DMFS (5I\% vs $30.2 \%, P<0.000 I)$ \\
\hline \multirow[t]{4}{*}{ EORTC $\left.2296\right|^{22}$} & Stage $\mathrm{T} 2 \mathrm{c}-\mathrm{T} 4$ & EBRT +6 months of triptorelin & 483 & Median follow-up: 6.4 years \\
\hline & Lymph node-positive & and flutamide or bicalutamide & & Long-term ADT improved at 5 years: \\
\hline & & EBRT +36 months of triptorelin and & 487 & PCSM (3.2\% vs $4.7 \%, P=0.002)$ \\
\hline & & 6 months of flutamide or bicalutamide & & \\
\hline \multirow[t]{9}{*}{ RTOG $92-02^{23}$} & Stage $\mathrm{T} 2 \mathrm{c}-\mathrm{T} 4$ & EBRT +4 months of goserelin & 763 & Median follow-up: II.3 years \\
\hline & $\mathrm{PSA}<150 \mathrm{ng} / \mathrm{mL}$ & and flutamide & & Long-term ADT improved at 10 years: \\
\hline & & EBRT +28 months of goserelin & 758 & PCSM (II.3\% vs $16.1 \%, P=0.0042)$ \\
\hline & & and 4 months of flutamide & & DFS $(22.5 \%$ vs I $3.2 \%, P<0.000 I)$ \\
\hline & & & & BF $(51.9 \%$ vs $68.1 \%, P<0.0001)$ \\
\hline & & & & DMFS (I4.8\% vs $22.8 \%, P<0.000 I)$ \\
\hline & & & & OS was only improved for patients \\
\hline & & & & with Gleason $8-10$ cancers $(45.1 \%$ vs \\
\hline & & & & $31.9 \%, P=0.0061)$ \\
\hline
\end{tabular}

Abbreviations: EORTC, European Organization for Research and Treatment of Cancer; EBRT, external beam radiation therapy; ADT, androgen deprivation therapy; OS, overall survival; PCSM, prostate cancer-specific mortality; DFS, disease-free survival; DMFS, distant metastases-free survival; RTOG, Radiation Therapy Oncology Group; PSA, prostate-specific antigen; BF, biochemical failure; WHO, World Health Organization.

high-grade (World Health Organization grade 3) or bulky (stage T3-T4) prostate cancer without evidence of metastatic disease to either EBRT with 36 months of ADT or to EBRT alone. ADT consisted of goserelin given every 28 days for 3 years. ${ }^{21}$ Goserelin was started on the first day of radiotherapy. Cyproterone acetate, a steroidal antiandrogen, was given for 1 month starting 1 week before goserelin. Ten-year overall survival was significantly improved in the ADT and EBRT arm compared with the EBRT alone arm $(58.1 \%$ vs $39.8 \%$, HR: $0.60, P=0.0004){ }^{21}$ There was also improvement in the prostate cancer-specific mortality (10.3\% vs $30.4 \%$, HR: 0.38 , $P<0.0001)$ and clinical disease-free survival $(47.7 \%$ vs $22.7 \%$, HR: $0.42, P<0.0001)$. The 10 -year locoregional failure rate was $6.0 \%$ in the ADT and EBRT arm vs $23.5 \%$ in the EBRT alone arm (HR: $0.21, P<0.0001$ ). There was also improvement in distant metastasis-free survival with the addition of ADT to EBRT (51\% vs 30.2\%, HR: 0.50, $P<0.0001)$.

EORTC 22961 randomized patients with locally advanced prostate cancer (stage T2c-T4) or lymph node-positive prostate cancer (T1 c-T4, N1 or N2) to either EBRT with 36 months of ADT or EBRT plus 6 months of ADT. ${ }^{22}$ The first 6 months of ADT consisted of a luteinizing hormone-releasing hormone (LHRH) agonist given every 28 or 84 days starting the first day of radiotherapy. A nonsteroidal antiandrogen (either flutamide $750 \mathrm{mg}$ per day or bicalutamide $50 \mathrm{mg}$ per day) was started 1 week before the LHRH agonist and continued for 6 months. Patients on the 36-month ADT arm were then treated for another 30 months with an LHRH agonist alone. The 5-year overall mortality was $15.2 \%$ for patients receiving 36 months of ADT vs $19.0 \%$ for the patients receiving 6 months of ADT. Five-year specific mortality was also improved in the 36-month group $(3.2 \%$ vs $4.7 \%, P=0.002){ }^{22}$

RTOG 92-02 was a follow-up study to RTOG $86-10$ and compared 4 months of ADT plus EBRT with 28 months of ADT plus EBRT. Patients with T2c-T4 prostate cancer and no evidence of metastatic disease were eligible for the study. ${ }^{23}$ ADT was given for 4 months using goserelin $3.6 \mathrm{mg}$ monthly and flutamide $250 \mathrm{mg}$ three times daily starting 2 months before radiotherapy. Patients randomized to the 28-month arm received an additional 24 months of goserelin alone. The 10-year disease-free survival was $22.5 \%$ for patients receiving EBRT with 28 months of ADT compared with $13.2 \%$ for the patients receiving EBRT with 4 months of ADT $(P<0.0001){ }^{23}$ In addition, there was a significant improvement in disease-specific survival ( $88.7 \%$ vs $83.9 \%, P=0.0042)$, biochemical failure $(51.9 \%$ vs $68.1 \%, P<0.0001)$, local progression $(12.3 \%$ vs $22.2 \%$, $P<0.0001)$, and distant metastasis $(14.8 \%$ vs $22.8 \%$, $P<0.0001)$ with long-term ADT. There was no significant difference in 10-year overall survival between long-term ADT and short-term ADT (53.9\% vs 51.6\%, $P=0.359$ ). When patients with a Gleason score of 8-10 were analyzed, there was a statistically significant improvement in disease-free survival $(20.8 \%$ vs $9.4 \%, P<0.001)$, overall 
survival $(45.1 \%$ vs $31.9 \%, P=0.0061)$, disease-specific survival (79.8\% vs $66.9 \%, P=0.0072)$, biochemical failure (56.0\% vs $73.9 \%, P<0.0001)$, local progression (17.8\% vs $27.3 \%, P=0.0338)$, and distant metastasis $(25.6 \%$ vs $39.7 \%$, $P=0.0019)$ with long-term ADT.

\section{GnRH agonist given with surgery}

There have been multiple randomized studies looking at the benefit of giving ADT before prostatectomy. To date, none of these studies have been able to show a statistically significant improvement in biochemical relapse-free survival with the addition of $\mathrm{ADT}^{24-27}$

ADT can be given after surgery to reduce the risk of recurrence. A study by Messing et al randomized patients who underwent prostatectomy and were found to have lymph node-positive disease to either immediate ADT or observation until there were signs of clinical progression other than a rising PSA. ${ }^{28}$ ADT consisted of either goserelin given every 28 days indefinitely or bilateral orchiectomy. Patients in the observation arm were treated with ADT and/or radiotherapy if they developed clinical evidence of recurrence. With a median follow-up of 11.9 years, the study showed significant improvement in overall survival (HR: $1.84, P=0.04$ ), prostate cancer-specific survival (HR: $4.09, P=0.0004$ ), and prostate cancer recurrence (HR: $3.42, P<0.0001$ ) for patients who were randomized to the immediate ADT arm. ${ }^{5}$ Of note, of the 36 patients in the observation group who received ADT, 34 (94.4\%) were started on ADT because they developed metastatic disease. Only two patients were started on ADT because of local recurrence.

\section{Decrease in size of prostate}

GnRH agonists may be used in patients with an enlarged prostate before radiotherapy. Whittington et al showed that there was a median decrease of $33 \%$ in prostate volume with 3-6 months of ADT using a GnRH agonist. ${ }^{29}$ Several other reports have shown the efficacy of ADT in reducing the size of the prostate, with the reduction in the range of $25 \%-55 \%{ }^{30,31}$ Such a reduction in size may be enough to allow a patient with significant pubic arch interference to undergo prostate seed brachytherapy. Decrease in the size of the prostate can also be beneficial for patients undergoing EBRT. Zelefsky et al showed that 3 months of leuprolide and flutamide prior to three-dimensional radiotherapy significantly decreased the radiation dose given to normal tissues. ${ }^{32}$ In 10 of 13 patients $(78 \%)$, there was a median reduction of $25 \%$ (range $16 \%-48 \%$ ) in the rectal volume receiving $95 \%$ of the prescription dose (D95). In 9 of 10 patients $(90 \%)$, there was a median reduction of $50 \%$ (range 6\%-64\%) in the bladder volume receiving D95. In 13 of 16 patients $(81 \%)$, there was a median reduction of $88 \%(67 \%-100 \%)$ of the small volume receiving D95. However, it should be noted that such a dose reduction seen on a dose-volume histogram may not lead to a decrease in toxicity. Pedersen et al showed that there was no obvious relationship between bladder dose-volume and risk of genitourinary toxicity. ${ }^{33}$

\section{Toxicity Decrease in quality of life}

Androgen deprivation can cause fatigue, hot flashes, decreased libido and erectile function, and gynecomastia, all of which can affect quality of life (QOL). Herr and O'Sullivan examined QOL in 79 men who were started on ADT for locally advanced disease or biochemical relapse after definitive treatment. ${ }^{34}$ In this study, men treated with ADT had worsening physical function, fatigue, and sexual function than patients undergoing observation or local therapy. van Andel and Kurth compared QOL in men with asymptomatic, lymph node-positive prostate cancer who were started on ADT vs controls. ${ }^{35}$ The baseline QOL evaluation was done 6 months after the diagnosis; however, it was not clear if patients were already started on ADT at this time. At baseline, patients on ADT had worse emotional function and overall QOL, but otherwise had general QOL domain scores similar to those of controls. Patients undergoing ADT had worse sexual function and more hot flashes. One year later, patients on ADT had worse physical function and fatigue; however, other general domains, including emotional function and overall QOL, were similar to the control patients. Sexual function and hot flashes remained worse in patients undergoing ADT. Alibhai et al enrolled 87 patients on ADT into a prospective study evaluating the effect of ADT on QOL. ${ }^{36}$ In addition, 86 patients with prostate cancer and 86 healthy patients were also enrolled. Endurance, upper and lower extremity strength, and QOL were evaluated at baseline, and at 3, 6, and 12 months. At baseline, patients in each group had similar performance on all three tests of physical function and scores on the QOL evaluation. Over time, patients in the ADT group had a decrease in grip strength and QOL compared with the two control groups $\left(P=0.04\right.$ and $P<0.001$, respectively). ${ }^{36}$ These changes were seen within 3 months of starting ADT and were generally independent of age. Of note, endurance remained stable for the ADT group, but improved in both control groups.

It may be possible to mitigate some of the adverse symptoms of ADT affecting QOL. Regular exercise may 
decrease the fatigue experienced with ADT. A study by Segal et al examining the use of exercise randomized 155 men receiving ADT to either resistance or aerobic exercise three times per week or usual care. ${ }^{37}$ Patients in the exercise arm experienced significantly less fatigue, a higher QOL, and improved muscular fitness. Medications (such as megesterol and venlafaxine) can be used to treat hot flashes. Gynecomastia can be prevented in patients by prophylactic radiotherapy to the breast tissue.

The effect on QOL is also dependent on duration of ADT. Patients receiving 6 months of ADT in EORTC 22961 were less likely to have insomnia $(P=0.006)$, hot flashes $(P<0.001)$, and reduced sexual interest and activity $(P<0.001)$ than patients receiving 36 months of treatment. ${ }^{22}$ Interestingly, the overall QOL did not differ significantly between the two groups $(P=0.37)$.

\section{Anemia}

ADT has been shown to cause a decline in hemoglobin in several studies. Fonseca et al showed a median hemoglobin decline of $1.2 \mathrm{~g} / \mathrm{dL}$ after bilateral orchiectomy. ${ }^{38}$ This decrease occurred within 90 days of surgery. Chander et al showed declines in hemoglobin of $1.01 \mathrm{~g} / \mathrm{dL}$ over 16 months of ADT with a GnRH agonist, with the greatest decline seen during the first 4 months. ${ }^{39}$ Hemoglobin levels increased slowly after the completion of ADT and corresponded to the increase in testosterone level. Of note, there were no correlations between the change in hemoglobin level and global QOL, physical function, or fatigue. A greater decrease in hemoglobin has been seen when a GnRH agonist is combined with a nonsteroidal antitestosterone. Strum et al showed a mean hemoglobin decrease of $2.54 \mathrm{~g} / \mathrm{dL}$ when patients were treated with combined androgen blockade. ${ }^{40}$

The clinical significance of ADT-induced anemia is uncertain at this time because the data show that the anemia is not correlated with symptoms and the hemoglobin does recover as the testosterone recovers. Patients should be tested with a blood count before starting ADT to make sure that they do not have anemia at baseline from other causes.

\section{Cognitive changes}

The effect of ADT on cognition is not clear at this time, especially since the patient population receiving ADT is already at risk of impaired cognition as a consequence of advancing age and medical comorbidities. Furthermore, studies analyzing cognitive decline with ADT show inconsistent results.

Green et al measured the effects of ADT on cognitive function in 82 men with advanced prostate cancer. ${ }^{41}$
These patients were randomized to one of three forms of medical castration or close monitoring. Nearly half of the men receiving ADT showed a statistically significant decline in one or more cognitive tests at 6 months, predominantly in tests of verbal memory and executive function. In contrast, none of the patients randomized to close monitoring showed a significant decline in any of the tests. Green et al then compared patients in the study with 20 healthy control patients. ${ }^{42}$ Once again, neither the patients undergoing close monitoring nor the healthy controls were found to have a significant decline in cognitive function. Jenkins et al showed a significant decline in 32 patients with localized prostate cancer undergoing short-term GnRH agonist therapy when compared with 18 healthy patients who were matched for age and intelligence. ${ }^{43}$ Spatial memory and spatial ability were significantly affected with the use of a GnRH agonist. When these patients were reassessed at 9 months after the completion of ADT, there was no significant difference between the two groups. Cherrier et al showed that there were no significant changes in verbal memory, spatial memory, executive function, or language between patients treated with ADT and healthy controls. ${ }^{44}$ Although there was a significant decline in spatial ability in patients undergoing ADT, there was an actual improvement in verbal memory. Such results make it difficult to determine if GnRH agonist use affects cognitive function. Even if GnRH agonist use causes decreased cognition, it is not known at this time if such a change would be clinically significant and affect day-to-day function or QOL.

\section{Obesity}

Because androgens preferentially promote lean body mass over fat mass, androgen deprivation can lead to a change in body habitus. Prospective trials have shown that in the first year of ADT, fat mass and weight can increase by $10 \%$ and $2 \%$, respectively, and lean body mass can decrease by $3 \% .{ }^{45-47}$ In fact, significant increases in fat mass can occur as soon as 3 months after the initiation of a GnRH agonist.

Imaging studies have shown that ADT can lead to increased accumulation of subcutaneous abdominal fat. ${ }^{46,48}$ Such an increase in abdominal girth is worrisome, because a large prospective cohort study has shown that abdominal circumference was strongly associated with mortality even after adjustment for body mass index. ${ }^{49}$

There have been very limited data on prevention or treatment of ADT-associated changes in body composition. A study examining the use of exercise randomized 155 men receiving ADT to either aerobic or resistance exercise three times 
per week or usual care. Although neither aerobic nor resistance exercise prevented the weight gain seen with ADT, resistance exercise helped patients avoid an increase in body fat. ${ }^{37}$

\section{Lipid alterations}

Along with changes in body composition, GnRH agonists can also cause changes in serum lipid profile. Treatment with GnRH agonists has been shown to increase triglycerides by $26 \%$ and total cholesterol by approximately $10 \% \cdot 46,49,50$ In addition, high-density lipoprotein increases by $8 \%-11 \%$. The net effect of these changes on the risk of cardiovascular disease is unknown at this time. However, in the general population, there is a continuous relationship between serum cholesterol and cardiovascular mortality. ${ }^{51,52}$ Diet and lifestyle changes are recommended as first-line interventions to achieve a target low-density lipoprotein (LDL) level. ${ }^{53}$ If these methods are unsuccessful, statin medications are recommended because several prospective studies have shown the benefit of statins in decreasing mortality from coronary artery disease. ${ }^{54-56}$

Selective estrogen receptor modulators have been tested for their effects on bone mineral density and fracture risk, but have also been found to affect serum lipids. In a randomized, placebo-controlled Phase III trial, treatment with toremifene led to decreases in total cholesterol, LDL, and triglyceride levels and an increase in high-density lipoproteins. ${ }^{57}$

\section{Insulin resistance and diabetes mellitus}

Prospective trials have shown that GnRH agonists decrease insulin sensitivity. ${ }^{49,58,59}$ This decrease in insulin sensitivity can be observed within 12 weeks of ADT initiation. Decreased insulin sensitivity and obesity are both associated with type 2 diabetes mellitus. Two large population-based studies have shown that GnRH agonist use is associated with an increased incidence of diabetes. The first study examined 73,196 men in the Surveillance, Epidemiology and End Results (SEER)-Medicare database aged 66 years or older diagnosed with locoregional prostate cancer. ${ }^{60}$ Thirty-six percent of the patients were treated with GnRH agonists and a further $7 \%$ underwent bilateral orchiectomies. With a median follow-up of 4.55 years, there was an increased risk of diabetes in patients receiving GnRH agonists. The adjusted HR for a new diagnosis of diabetes was $1.44(P<0.001){ }^{60}$ The adjusted HR of patients undergoing orchiectomy for a new diagnosis of diabetes was $1.34(P<0.001)$. The second study examined 19,079 patients in a database from Ontario, Canada, aged 66 years or older treated for 6 months or more with a GnRH agonist or bilateral orchiectomies. ${ }^{61}$ When these patients were matched with men who were not treated with ADT, a statistically significant association between ADT and increased risk for diabetes (HR: 1.16, 95\% confidence interval [CI]: $1.11-1.21){ }^{61}$

Because of the observed greater risk for diabetes seen in these two analyses, patients undergoing ADT should undergo screening for diabetes. Patients can be tested for diabetes with either fasting plasma glucose and/or hemoglobin A1c levels. Patients at high risk of developing diabetes with an elevated fasting plasma glucose level (100-125 mg/dL) or an elevated hemoglobin A1c level (6.0\%-6.5\%) should be counseled according to the guidelines from the American Diabetes Association. ${ }^{53}$ Recommendations for screening and treatment are summarized in Table 4.

Table 4 Recommendations for screening and treatment of metabolic adverse effects ${ }^{53}$

\section{Hyperlipidemia and CAD}

Screening:

- Fasting lipid panel at baseline, after I year of deprivation therapy, and then as clinically indicated (use lipid levels defined in the NCEP ATP III to assign risk)

- Identify presence of clinical atherosclerotic disease that confers high risk for CAD

- Determine presence of major risk factors (other than LDL)

Treatment:

- Tobacco cessation for all

- Treatment of hypertension per American Heart Association guidelines

- TLC if LDL is above goal

- TLC diet

- Saturated fat $<7 \%$ of calories, cholesterol $<200 \mathrm{mg} /$ day

- Consider increased viscous (soluble) fiber (I0-25 g/day) and plant stanols/sterols ( $2 \mathrm{~g} /$ day) as therapeutic options to enhance LDL lowering

- Weight management

- Increased physical activity

- Identify metabolic syndrome and treat, if present, after 3 months of TLC - Consider adding drug therapy to treat lipid levels

- If patient already has CAD or CAD equivalent

- If patients have a high triglyceride level

- Use aspirin for CAD patients to reduce prothrombotic state

Diabetes

Screening:

- Fasting glucose or hemoglobin Alc at baseline and then annually

- Diabetes: hemoglobin Alc $=6.5 \%$ or fasting glucose $=126 \mathrm{mg} / \mathrm{dL}$

- High risk of developing diabetes (prediabetes): hemoglobin Alc $6.0 \%-6.5 \%$ or fasting glucose $100-125 \mathrm{mg} / \mathrm{dL}$

Treatment of high-risk patients:

- Identify and treat other CAD risk factors

- Repeat testing at least annually and counsel lifestyle interventions (with follow-up counseling)

- $5 \%-10 \%$ weight loss

$-\geq 150$ minutes of moderate physical activity per week

Adapted from Saylor and Smith, Reprinted with permission from JNCCN-Journal of the National Comprehensive Cancer Network.

Abbreviations: CAD, coronary artery disease; LDL, low-density lipoprotein; TLC, therapeutic lifestyle changes; NCEP ATP III, National Cholesterol Education Program Adult Treatment Panel III. 


\section{Coronary artery disease and myocardial infarction}

As stated earlier, GnRH agonists cause elevations in triglycerides and cholesterol, weight gain, and insulin resistance. GnRH agonists have also been shown to increase the risk of diabetes in two large population-based studies. Such a combination of side effects should lead to an elevated risk of coronary artery disease; however, the available data show conflicting results.

A large SEER-Medicare-based analysis of 73,196 men aged 66 years or older with prostate cancer showed a significantly increased risk of a new diagnosis of coronary artery disease (HR: 1.16, $P<0.001$ ), myocardial infarction (HR: $1.11, P=0.03$ ), and sudden cardiac death (HR: 1.16, $P=0.004)$ with the use of GnRH agonists. ${ }^{60}$ Interestingly, patients who underwent orchiectomy were not at increased risk of cardiovascular morbidity or mortality. A second SEER-Medicare-based study of 22,816 men confirmed the results, showing a $20 \%$ increase in the risk of cardiovascular morbidity at 1 year with ADT. ${ }^{62}$ In contrast, a study of 19,079 men aged 66 years or older from Ontario did not show any correlation between ADT use and acute myocardial infarction (HR: $0.91,95 \%$ CI: $0.84-1.00$ ) or sudden cardiac death (HR: 0.96, 95\% CI: 0.83-1.10) ${ }^{61}$ Another study was performed using the Cancer of the Prostate Strategic Urologic Research Endeavor (CAPSURE) database, looking at 3262 men treated with prostatectomy and 1630 patients treated with EBRT, brachytherapy, or cryotherapy for their localized prostate cancer. ${ }^{63}$ This study showed that ADT was significantly associated with an increased risk of death from cardiovascular causes in patients treated with prostatectomy (HR: $2.6,95 \% \mathrm{CI}: 1.4-4.7, P=0.002$ ). This increased risk was greater for patients aged 65 years or older. ADT use was associated with a higher incidence of death from cardiovascular causes in men aged 65 years or older who were treated with EBRT, brachytherapy, or cryotherapy, but the difference did not reach statistical significance.

As stated earlier, there have been several randomized trials to date studying the benefit of ADT in patients undergoing EBRT. D'Amico et al analyzed 1372 patients enrolled into three randomized trials ( 0 vs 3,3 vs 8 , or 0 vs 6 months of ADT) for increased risk of fatal myocardial infarction. ${ }^{64}$ In this analysis, there was no increased incidence of fatal myocardial infarction seen. However, men aged 65 years or older who received 6 months of ADT experienced shorter times of fatal myocardial infarction compared with men who did not receive ADT. Interestingly, D'Amico et al also showed that adding 6 months of ADT to EBRT only benefitted patients who did not have moderate or severe medical comorbidities based on the Acute Comorbidity Evaluation 27, which suggests that the potential harm of ADT may outweigh the potential benefit in this group of patients. ${ }^{17}$ Both RTOG 86-10 and RTOG 92-02 have been analyzed retrospectively for an association between ADT and cardiovascular mortality. ${ }^{18,65}$ These analyses did not show any significant association. EORTC 22863 has also been retrospectively analyzed; once again, there was no significant association seen between ADT use and cardiovascular mortality. ${ }^{22}$

Although the available data are inconsistent, it is probably prudent to make sure that patients on ADT try to minimize their risk of coronary artery disease by following guidelines as recommended by the National Cholesterol Education Program Adult Treatment Panel III and the American Heart Association. ${ }^{53}$ Recommendations for screening and treatment are summarized in Table 4.

\section{Osteoporosis and fracture risk}

ADT has been associated with accelerated bone turnover, decreased bone mineral density, and increased risk of fractures. ${ }^{66-68}$ A study of 50,613 men in the SEER-Medicare database showed an increase in the incidence of fractures 5 years after the diagnosis of prostate cancer from $12.6 \%$ to $19.4 \%$ with ADT. ${ }^{69}$ Another study using claims data showed a significant association between GnRH agonist use and clinical fractures (relative risk [RR]: $1.21, P<0.001$ ). ${ }^{70}$

Bisphosphonates, including pamidronate, ${ }^{68,71}$ alendronate, ${ }^{72}$ risedronate, ${ }^{73}$ and zoledronic acid, ${ }^{74,75}$ have all been found to increase bone mineral density in patients undergoing ADT. However, these studies have not been able to show a significant decrease in the incidence of treatmentrelated fractures. In contrast, there are two additional classes of medications, which have been shown to decrease the risk of fractures in patients undergoing ADT.

Receptor activator of nuclear factor kappa-B ligand (RANKL) regulates the differentiation, function, and survival of osteoclasts (which mediate bone breakdown). ${ }^{76}$ Denosumab, a human monoclonal antibody against RANKL, was studied in a randomized, placebo-controlled Phase III trial. The study enrolled 1468 patients receiving ADT and at high risk of fracture (defined as having a history of fracture, age 70 years or older, or low bone mineral density). ${ }^{77}$ When given subcutaneously every 6 months, denosumab increased bone mineral density at all measured sites and reduced the incidence of new vertebral fractures by $62 \%(P=0.006)$ and multiple fractures at any site by $62 \%(P=0.006)$. There were a decrease of $28 \%$ in the incidence of fractures at any site, 
Table 5 Recommendations for screening and treatment of osteoporosis $^{53}$

Screening:

- Test bone mineral density at baseline, repeat after I year of ADT, and repeat as clinically indicated

- Consider using WHO/FRAX fracture risk assessment tool

Treatment:

- Supplemental calcium ( $\geq 1200 \mathrm{mg} /$ day) and vitamin D (800-1000 IU/day) for all

- Consideration of drug treatment if age $\geq 50$ years and any of the following:

- Personal history of hip or vertebral fracture

- $\mathrm{T}$-score $=-2.5$ at the femoral neck or spine

- Low T-score at femoral neck or spine (-1.0 to -2.5$)$ and either of the following US-adapted WHO algorithm

- 10-year probability of a hip fracture $\geq 3 \%$ or

- I0-year probability of a major osteoporosis-related fracture $\geq 20 \%$

Adapted from Saylor and Smith, Reprinted with permission from JNCCN-Journal of the National Comprehensive Cancer Network.

Abbreviations: ADT, androgen deprivation therapy; WHO, World Health Organization.

but this was not statistically significant $(P=0.10)$. Selective estrogen receptor modulators have also been shown to be effective in decreasing fractures in patients receiving ADT. Toremifene was studied in a randomized, placebo-controlled Phase III trial enrolling 1389 patients aged 50 years or older treated with ADT who were at increased risk of fracture (age 70 years or older or low bone mineral density). ${ }^{78}$ Patients on the toremifene arm experienced fewer vertebral fractures (2.5\% vs $4.9 \%$, RR: $0.50, P<0.05)$.

At this time, screening and treatment recommendations should be extrapolated from the National Osteoporosis Foundation guidelines for the general population..$^{53}$ Patients on ADT should be considered at high risk for developing osteoporosis and increased risk for fractures. All men 50 years or older should be encouraged to take supplemental calcium ( $\geq 1200 \mathrm{mg}$ per day) and vitamin D (800-1000 IU per day). Bisphosphonates should be used in patients with osteoporosis ( $\mathrm{T}$ score $\leq-2.5$ on bone density scan) or patients with a previous history of hip or vertebral body fracture. These recommendations are summarized in Table 5.

\section{Conclusion}

ADT has been shown to have a significant benefit in the treatment of prostate cancer. ADT can be used with EBRT to improve clinical outcome. ADT has been shown to improve overall survival in patients with lymph node-positive disease who underwent prostatectomy and lymph node dissection. Finally, ADT is the first-line treatment for patients with metastatic cancer, and has an approximately $85 \%$ response rate. Prostate cancer-specific mortality is relatively low in most patients, and even men with metastatic prostate cancer may live for years. Therefore, it is crucial that the benefits of ADT be weighed against the potential adverse effects. ADT can cause constitutional symptoms (such as fatigue and hot flashes) and decreased libido. The clinical significance of ADT-induced anemia and the effect of ADT on cognition are not well established at this time. ADT can cause changes in body composition and development of insulin resistance, with progression to diabetes mellitus. Practice guidelines have been developed for the screening and treatment of diabetes. The association between ADT and coronary artery disease is somewhat less clear; however, patients should be monitored closely due to the changes in lipid profile with ADT. Patients undergoing ADT are also at increased risk for osteoporosis and fractures. Patients should be evaluated and treated based on the National Osteoporosis Foundation guidelines. Although significant advances have been made to date, further clinical investigation is needed to identify better the patients at risk of developing ADT-related side effects and to define more effective treatments.

\section{Disclosure}

The authors report no conflicts of interest in this work.

\section{References}

1. American Cancer Society. Facts and Figures 2010. Available from: http:// www.cancer.org/Research/CancerFactsFigures/CancerFactsFigures/ cancer-facts-and-figures-2010. Accessed October 28, 2011.

2. The Leuprolide Study Group. Leuprolide versus diethylstilbestrol for metastatic prostate cancer. N Engl J Med. 1984;311:1281-1286.

3. Vogelzang NJ, Chodak GW, Soloway MS, et al. Goserelin versus orchiectomy in the treatment of advanced prostate cancer: final results of a randomized trial. Urology. 1995;46:220-226.

4. The Medical Research Council Prostate Cancer Working Party Investigators Group. Immediate versus deferred treatment for advanced prostatic cancer: initial results of the Medical Research Council trial. Br J Urol. 1997;79:235-246.

5. Messing EM, Manola J, Yao J, et al. Immediate versus deferred androgen deprivation treatment in patients with node-positive prostate cancer after radical prostatectomy and pelvic lymphadenectomy. Lancet Oncol. 2006; 7:472-479.

6. Kelleher S, Conway AJ, Handelsman DJ. Blood testosterone threshold for androgen deficiency symptoms. J Clin Endocrinol Metab. 2004;89: 3813-3817.

7. Zitzmann M, Faber S, Nieschlag E. Association of specific symptoms and metabolic risks with serum testosterone in older men. $J$ Clin Endocrinol Metab. 2006;91:4335-4343.

8. Chon J, Jacobs S, Naslund M. The cost value of medical versus surgical hormonal therapy for metastatic prostate cancer. J Urol. 2000;164: 735-737.

9. Conn PM, Crowley WF Jr. Gonadotropin-releasing hormone and its analogs. Annu Rev Med. 1994;45:391-405.

10. Klotz L, Boccon-Gibod L, Shore ND, et al. The efficacy and safety of degarelix: a 12-month, comparative, randomized, open-label, parallelgroup Phase III study in patients with prostate cancer. BJU Int. 2008; 102:1531-1538.

11. de Bono JS, Logothetis CJ, Molina A, et al. Abiraterone and increased survival in metastatic prostate cancer. N Engl J Med. 2011;364: 1995-2005. 
12. Page ST, Lin DW, Mostaghel EA, et al. Persistent intraprostatic androgen concentrations after medical castration in healthy men. J Clin Endocrinol Metab. 2006;91:3850-3856.

13. Gao W, Bohl CE, Dalton JT. Chemistry and structural biology of androgen receptor. Chem Rev. 2005;105:3352-3370.

14. Tran C, Ouk S, Clegg NJ, et al. Development of a second-generation antiandrogen for treatment of advanced prostate cancer. Science. 2009;324:787-790.

15. Shipley WU, Hunt D, Lukka H, et al. Initial report of RTOG 9601, a phase III trial in prostate cancer: effect of anti-androgen therapy (AAT) with bicalutamide during and after radiation therapy (RT) on freedom from progression and incidence of metastatic disease in patients following radical prostatectomy (RP) with pT2-3, N0 disease and elevated PSA levels. Paper presented at American Society of Clinical Oncology Genitourinary Cancers Symposium, February 17-19, 2011, Orlando, FL.

16. Klotz L, O'Callaghan J, Ding K, et al. A phase III randomized trial comparing intermittent versus continuous androgen suppression for patients with PSA progression after radical therapy: NCIC CTG PR.7/ SWOG JPR.7/CTSU JPR.7/UK Intercontinental Trial CRUKE/01/013. Paper presented at American Society of Clinical Oncology Genitourinary Cancers Symposium, February 17-19, 2011, Orlando, FL.

17. D'Amico AV, Chen M-H, Renshaw AA, et al. Androgen suppression and radiation vs radiation alone for prostate cancer. JAMA. 2008; 299:289-295

18. Roach M, Bae K, Speight J, et al. Short-term neoadjuvant androgen deprivation therapy and external-beam radiotherapy for locally advanced prostate cancer: long-term results of RTOG 8610. J Clin Oncol. 2008;26:585-591.

19. Jones CU, Hunt D, McGowan DG, et al. Radiotherapy and short-term androgen deprivation for localized prostate cancer. $N$ Engl J Med. 2011; 365:107-118.

20. Denham JW, Steigler A, Lamb DS, et al. Short-term neoadjuvant androgen deprivation and radiotherapy for locally advanced prostate cancer: 10-year data from the TROG 96.01 randomised trial. Lancet Oncol. 2011;12:451-459.

21. Bolla M, Van Tienhoven G, Warde P, et al. External irradiation with or without long-term androgen suppression for prostate cancer with high metastatic risk: 10-year results of an EORTC randomised study. Lancet Oncol. 2010;11:1066-1073.

22. Bolla M, de Reijke TM, Van Tienhoven G, et al. Duration of androgen suppression in the treatment of prostate cancer. NEngl J Med. 2009;360: 2516-2527.

23. Horwitz EM, Bae K, Hanks GE, et al. Ten-year follow-up of Radiation Therapy Oncology Group Protocol 92-02: a Phase III trial of the duration of elective androgen deprivation in locally advanced prostate cancer. J Clin Oncol. 2008;26:2497-2504.

24. Aus G, Abrahamsson PA, Ahlgren G, et al. Three-month neoadjuvant hormonal therapy before radical prostatectomy: a 7-year follow-up of a randomized controlled trial. BJU Int. 2002;90:561-566.

25. Klotz LH, Goldenberg SL, Jewett MAS, et al. Long-term followup of a randomized trial of 0 versus 3 months of neoadjuvant androgen ablation before radical prostatectomy. J Urol. 2003;170:791-794.

26. Schulman C, Debruyne F, Forster G, et al. 4-year follow-up results of a European prospective randomized study on neoadjuvant hormonal therapy prior to radical prostatectomy in T2-3N0M0 prostate cancer. European Study Group On Neoadjuvant Treatment Of Prostate Cancer. Eur Urol. 2000;38:706-713.

27. Soloway MS, Pareek K, Sharifi R, et al. Neoadjuvant androgen ablation before radical prostatectomy in cT2bNxM0 prostate cancer: 5-year results. J Urol. 2002;167:112-116.

28. Messing EM, Manola J, Sarosdy M, et al. Immediate hormonal therapy compared with observation after radical prostatectomy and pelvic lymphadenectomy in men with node-positive prostate cancer. $N$ Engl J Med. 1999;341:1781-1788.

29. Whittington R, Broderick GA, Arger P, et al. The effect of androgen deprivation on the early changes in prostate volume following transperineal ultrasound guided interstitial therapy for localized carcinoma of the prostate. Int J Radiat Oncol Biol Phys. 1999;44:1107-1110.
30. Shearer R, Davies J, Gelister J, et al. Hormonal cytoreduction and radiotherapy for carcinoma of the prostate. Br J Urol. 1992;69:521-524.

31. Sneller Z, Hop W, Carpentier P, et al. Prognosis and prostatic volume changes during endocrine management of prostate cancer: a longitudinal study. J Urol. 1992;147:962-966.

32. Zelefsky M, Leibel S, Burman C, et al. Neoadjuvant hormonal therapy improves the therapeutic ratio in patients with bulky prostatic cancer treated with three-dimensional conformal radiation therapy. Int J Radiat Oncol Biol Phys. 1994;29:755-761.

33. Pederson AW, Fricano J, Correa D, Pelizzari CA, Liauw SL. Late toxicity after intensity-modulated radiation therapy for localized prostate cancer: an exploration of dose-volume histogram parameters to limit genitourinary and gastrointestinal toxicity. Int J Radiat Oncol Biol Phys. 2011. [Epub ahead of print.]

34. Herr HW, O'Sullivan M. Quality of life of asymptomatic men with nonmetastatic prostate cancer on androgen deprivation therapy. J Urol. 2000;163:1743-1746.

35. van Andel G, Kurth KH. The impact of androgen deprivation therapy on health related quality of life in asymptomatic men with lymph node positive prostate cancer. Eur Urol. 2003;44:209-214.

36. Alibhai SMH, Breunis H, Timilshina $\mathrm{N}$, et al. Impact of androgendeprivation therapy on physical function and quality of life in men with nonmetastatic prostate cancer. J Clin Oncol. 2010;28:5038-5045.

37. Segal RJ, Reid RD, Courneya KS, et al. Randomized controlled trial of resistance or aerobic exercise in men receiving radiation therapy for prostate cancer. J Clin Oncol. 2009;27:344-351.

38. Fonseca R, Rajkumar S, White W, et al. Anemia after orchiectomy. Am J Hematol. 1998;59:230-233.

39. Chander S, Choo R, Danjoux C, et al. Effect of androgen suppression on hemoglobin in prostate cancer patients undergoing salvage radiotherapy plus 2-year buserelin acetate for rising PSA after surgery. Int J Radiat Oncol Biol Phys. 2005;62:719-724.

40. Strum S, McDermed J, Scholz M, et al. Anaemia associated with androgen deprivation in patients with prostate cancer receiving combined hormone blockade. Br J Urol. 1997;79:933-941.

41. Green HJ, Pakenham KI, Headley BC, et al. Altered cognitive function in men treated for prostate cancer with luteinizing hormone-releasing hormone analogues and cyproterone acetate: a randomized controlled trial. BJU Int. 2002;90:427-432.

42. Green HJ, Pakenham KI, Headley BC, et al. Quality of life compared during pharmacological treatments and clinical monitoring for nonlocalized prostate cancer: a randomized controlled trial. BJU Int. 2004; 93:975-979.

43. Jenkins VA, Bloomfield DJ, Shilling VM, et al. Does neoadjuvant hormone therapy for early prostate cancer affect cognition? Results from a pilot study. BJU Int. 2005;96:48-53.

44. Cherrier MM, Rose AL, Higano C. The effects of combined androgen blockade on cognitive function during the first cycle of intermittent androgen suppression in patients with prostate cancer. J Urol. 2003;170: 1808-1811.

45. Smith MR. Changes in fat and lean body mass during androgendeprivation therapy for prostate cancer. Urology. 2004;63:742-745.

46. Smith MR, Finkelstein JS, McGovern FJ, et al. Changes in body composition during androgen deprivation therapy for prostate cancer. J Clin Endocrinol Metab. 2002;87:599-603.

47. Smith MR, Lee H, McGovern F, et al. Metabolic changes during gonadotropin-releasing hormone agonist therapy for prostate cancer. Cancer. 2008;112:2188-2194.

48. Smith MR, Lee H, Fallon MA, et al. Adipocytokines, obesity, and insulin resistance during combined androgen blockade for prostate cancer. Urology. 2008;71:318-322.

49. Dockery F, Bulpitt CJ, Agarwal S, et al. Testosterone suppression in men with prostate cancer leads to an increase in arterial stiffness and hyperinsulinaemia. Clin Sci. 2003;104:195-201.

50. Eri LM, Urdal P, Bechensteen AG. Effects of the luteinizing hormonereleasing hormone agonist leuprolide on lipoproteins, fibrinogen and plasminogen activator inhibitor in patients with benign prostatic hyperplasia. J Urol. 1995;154:100-104. 
51. Lewington S, Whitlock G, Clarke R, et al. Blood cholesterol and vascular mortality by age, sex, and blood pressure: a meta-analysis of individual data from 61 prospective studies with 55,000 vascular deaths. Lancet. 2007;370:1829-1839.

52. Stamler J, Wentworth D, Neaton J. Is relationship between serum cholesterol and risk of premature death from coronary heart disease continuous and graded? Findings in 356,222 primary screenees of the Multiple Risk Factor Intervention Trial (MRFIT). JAMA. 1986;28:2823-2828.

53. Saylor P, Smith M. Adverse effects of androgen deprivation therapy: defining the problem and promoting health among men with prostate cancer. J Natl Compr Canc Netw. 2010;8:211-223.

54. Sacks FM, Pfeffer MA, Moye LA, et al. The effect of pravastatin on coronary events after myocardial infarction in patients with average cholesterol levels. N Engl J Med. 1996;335:1001-1009.

55. Scandinavian Simvastatin Survival Study Group. Randomised trial of cholesterol lowering In 4444 patients with coronary heart disease: The Scandinavian Simvastatin Survival Study (4S). Lancet. 1994; 344:1383-1389.

56. The Long-Term Intervention with Pravastatin in Ischaemic Disease (LIPID) Study Group. Prevention of cardiovascular events and death with pravastatin in patients with coronary heart disease and a broad range of initial cholesterol levels. N Engl J Med. 1998;339:1349-1357.

57. Smith MR, Malkowicz SB, Chu F, et al. Toremifene improves lipid profiles in men receiving androgen-deprivation therapy for prostate cancer: interim analysis of a multicenter Phase III study. J Clin Oncol. 2008;26:1824-1829.

58. Smith JC, Bennett S, Evans LM, et al. The effects of induced hypogonadism on arterial stiffness, body composition, and metabolic parameters in males with prostate cancer. J Clin Endocrinol Metab. 2001 86:4261-4267.

59. Smith MR, Lee H, Nathan DM. Insulin sensitivity during combined androgen blockade for prostate cancer. J Clin Endocrinol Metab. 2006;91:1305-1308

60. Keating NL, O'Malley AJ, Smith MR. Diabetes and cardiovascular disease during androgen deprivation therapy for prostate cancer. J Clin Oncol. 2006;24:4448-4456.

61. Alibhai SMH, Duong-Hua M, Sutradhar R, et al. Impact of androgen deprivation therapy on cardiovascular disease and diabetes. J Clin Oncol. 2009;27:3452-3458

62. Saigal CS, Gore JL, Krupski TL, et al. Androgen deprivation therapy increases cardiovascular morbidity in men with prostate cancer. Cancer. 2007;110:1493-1500.

63. Tsai HK, D’Amico AV, Sadetsky N, et al. Androgen deprivation therapy for localized prostate cancer and the risk of cardiovascular mortality. J Natl Cancer Inst. 2007;99:1516-1524.

64. D'Amico AV, Denham JW, Crook J, et al. Influence of androgen suppression therapy for prostate cancer on the frequency and timing of fatal myocardial infarctions. J Clin Oncol. 2007;25:2420-2425.
65. Efstathiou JA, Bae K, Shipley WU, et al. Cardiovascular mortality and duration of androgen deprivation for locally advanced prostate cancer: Analysis of RTOG 92-02. Eur Urol. 2008;54:816-824.

66. Berruti A, Dogliott L, Terrone C, et al. Changes in bone mineral density, lean body mass and fat content as measured by dual energy $\mathrm{x}$-ray absorptiometry in patients with prostate cancer without apparent bone metastases given androgen deprivation therapy. J Urol. 2002; 167:2361-2367.

67. Maillefert JF, Sibilia J, Michel F, et al. Bone mineral density in men treated with synthetic gonadotropin-releasing hormone agonists for prostatic carcinoma. J Urol. 1999;161:1219-1222.

68. Smith MR, McGovern FJ, Zietman AL, et al. Pamidronate to prevent bone loss during androgen-deprivation therapy for prostate cancer. N Engl J Med. 2001;345:948-955

69. Shahinian VB, Kuo Y-F, Freeman JL, et al. Risk of fracture after androgen deprivation for prostate cancer. $N$ Engl J Med. 2005;352: 154-164.

70. Smith MR, Lee WC, Brandman J, et al. Gonadotropin-releasing hormone agonists and fracture risk: a claims-based cohort study of men with nonmetastatic prostate cancer. J Clin Oncol. 2005;23:7897-7903.

71. Diamond TH, Winters J, Smith A, et al. The antiosteoporotic efficacy of intravenous pamidronate in men with prostate carcinoma receiving combined androgen blockade. Cancer. 2001;92:1444-1450.

72. Greenspan S, Nelson J, Trump D, et al. Effect of once-weekly oral alendronate on bone loss in men receiving androgen deprivation therapy for prostate cancer: a randomized trial. Ann Intern Med. 2007;146: 416-424.

73. Izumi K, Mizokami A, Sugimoto K, et al. Risedronate recovers bone loss in patients with prostate cancer undergoing androgen-deprivation therapy. Urology. 2009;73:1342-1346.

74. Michaelson MD, Kaufman DS, Lee H, et al. Randomized controlled trial of annual zoledronic acid to prevent gonadotropin-releasing hormone agonist-induced bone loss in men with prostate cancer. J Clin Oncol. 2007;25:1038-1042.

75. Smith MR, Eastham J, Gleason DM, et al. Randomized controlled trial of zoledronic acid to prevent bone loss in men receiving androgen deprivation therapy for nonmetastatic prostate cancer. J Urol. 2003;169: 2008-2012.

76. Lacey DL, Timms E, Tan HL, et al. Osteoprotegerin ligand is a cytokine that regulates osteoclast differentiation and activation. Cell. 1998;93:165-176.

77. Smith MR, Egerdie B, Toriz NH, et al. Denosumab in men receiving androgen-deprivation therapy for prostate cancer. N Engl J Med. 2009; 361:745-755.

78. Smith MR, Malkowicz SB, Chu F, et al. Toremifene increases bone mineral density in men receiving androgen deprivation therapy for prostate cancer: interim analysis of a multicenter Phase 3 clinical study. J Urol. 2008;179:152-155.
Drug, Healthcare and Patient Safety

\section{Publish your work in this journal}

Drug, Healthcare and Patient Safety is an international, peer-reviewed open-access journal exploring patient safety issues in the healthcare continuum from diagnostic and screening interventions through to treatment, drug therapy and surgery. The journal is characterized by the rapid reporting of reviews, original research, clinical, epidemiological and

\section{Dovepress}

post-marketing surveillance studies, risk management, health literacy and educational programs across all areas of healthcare delivery. The manuscript management system is completely online and includes a very quick and fair peer-review system. Visit http://www.dovepress.com/ testimonials.php to read real quotes from published authors. 